\title{
De la rinitis al asma: ¿una o dos enfermedades?
}

\section{From rhinitis to asthma, one or two diseases?}

\section{M.J. Álvarez, J.M. Olaguíbel, E. Lasa, E. Arroabarren, A. Gómez, B. Gómez}

\section{RESUMEN}

La frecuente asociación entre la rinitis y el asma alérgicos puede ser consecuencia de su mera coexistencia en el tiempo. También es posible que ambas entidades sean los polos opuestos de una ambas entidades sean los polos opuestos de una misma enfermedad que afecta al aparato respiratorio o sistémica caracterizada por la respuesta inmunológica anómala frente al alergeno. Las interacciones entre fosas nasales y vías aéreas bronquiales han sido ampliamente estudiadas desde el aspecto epidemiológico, fisiopatológico, etiológico epidemiologico, fisiopatológico, etiológico y farmacologico. Los datos existentes a este respecto y

Palabras clave. Rinitis. Asma, Alergeno Mecanismos. Interacción nariz-pulmón.

\section{ABSTRACT}

Rhinitis and asthma commonly coexist in allergic patients. This observation might be attributable to their coexistence in time, but it is also possible that both diseases are the opposite poles of a single disease affecting the respiratory system, or even an anomalous systemic immune response to the allergen. Nose-lung systemic immune response to the allergen. Nose-lung interactions have been widely studied from epidemiological, physiopathological, aetiological and
pharmacological aspects. In the present article we pharmacological aspects. In the present article
review this topic and its main clinical implications.

Key Words. Rhinitis. Asthma. Allergen Mechanisms. Nose-lung interaction.

An. sis. sanit. Navar. 2003; 26 (Supl. 2): 49-55.

Sección de Alergología. Hospital Virgen del Camino. Pamplona

\author{
Correspondencia: \\ M.J. Álvarez Puebla \\ S. de Alergología \\ CS Conde Oliveto \\ Plaza de la Paz SN. Pamplona \\ Tfno. 948429303 \\ malvarpu@cfnavarra.es
}




\section{INTRODUCCIÓN}

La asociación entre rinitis y asma es bien conocida desde los tiempos antiguos y ya Galeno en el siglo II recomendaba "purgar las fosas nasales de secreciones con el fin de aliviar los pulmones" ${ }^{\prime}$. Durante los últimos años, la especialización de la medicina y la disponibilidad de fármacos órgano específicos, entre otros, determinaron que la rinitis y el asma fueran contempladas y tratadas como enfermedades diferentes, lo que se traduce en guías de diagnóstico y tratamiento individuales para cada una de ellas ${ }^{2,3}$.

Sin embargo, fosas nasales, nasofaringe, tráquea y pulmones están anatómicamente contiguas lo que probablemente determina su frecuente afectación simultánea. Un ejemplo de ello son las infecciones de vías aéreas superiores en las que los síntomas iniciales de rinitis con frecuencia se siguen de traqueobronquitis. La rinitis y el asma alérgicos, el síndrome de Samter (poliposis nasal, asma bronquial intrínseco e idiosincrasia a aspirina) o la fibrosis quística son ejemplos de esta asociación (Tabla 1). Esto, unido a la constatación de que el control subóptimo de los síntomas nasales es una de las causas más frecuentes del asma de mala evolución ${ }^{4}$ ha originado la hipótesis de la "vía aérea única" que sostiene que ambas entidades son los polos opuestos de una única enfermedad cuya base es el proceso inflamatorio crónico de la vía aérea que puede ser mantenido y amplificado por mecanismos interconectados. Las observaciones epidemiológicas, fisiopatológicas, etiológicas y farmacológicas que fundamentan esta teoría y sus implicaciones prácticas se revisan a continuación (Tabla 2).

\section{Epidemiología}

Es frecuente observar, tanto en la práctica clínica como en estudios epidemiológicos, la coexistencia de la rinitis y el asma alérgicos. Así, un estudio realizado en 475 adultos jóvenes demostró que un $98 \%$ de los sujetos diagnosticados de asma tenían también síntomas de rinitis y a la inversa, que el $78 \%$ de los individuos con rinitis, también mostraban síntomas de asma ${ }^{5}$. Las dos entidades muestran además similitudes en su origen, mecanis-

Tabla 1. Síndromes que asocian patología nasal y bronquial.

- Infecciones respiratorias.

- Rinitis y asma alérgicos.

- Síndrome de Samter (poliposis nasal, asma bronquial intrínseco, intolerancia a aspirina).

- Fibrosis quística.

- Discinesia ciliar.

- Síndrome de Wegener.

- Síndrome de Churg-Strauss.

- Aspergilosis broncopulmonar alérgica.

Tabla 2. Aspectos que sugieren el origen común de la rinitis y el asma alérgicos.

- Asociación clínica frecuente entre rinitis y asma

- La rinitis es un factor de riesgo independiente para el desarrollo de asma.

- Origen común: respuesta inmune anómala frente al alergeno.

- En la rinitis alérgica se aprecia eosinofilia bronquial y a la inversa.

- Algunos sujetos con rinitis alérgica presentan HRB.

- La provocación bronquial y la aplicación nasal del alergeno inducen en sujetos con rinitis una respuesta bronquial similar a la de asmáticos (eosinofilia pulmonar e incremento de la HRB).

- El tratamiento aislado del asma mejora los síntomas nasales y a la inversa.

- El control ambiental y la vacunación con alergeno mejoran ambas entidades e interfieren la progresión natural de la enfermedad (desarrollo de asma y de nuevas sensibilizaciones). 
mo fisiopatológico e historia natural ya que con frecuencia, la rinitis precede al asma, considerándose como un factor de riesgo independiente para su desarrollo ${ }^{6}$. De acuerdo con ello, se ha constatado que los sujetos con rinitis perenne tienen 11 veces más riesgo de desarrollar asma que la población general.

\section{Fisiopatología}

La respuesta inmunoalérgica que experimenta la mucosa respiratoria es idéntica en vías aéreas superiores e inferiores, apreciándose inicialmente una reacción de hipersensibilidad IgE-mediada, específica para el alergeno que desencadena la liberación de mediadores preformados por mastocitos. Esta reacción se sigue de un reclutamiento de linfocitos colaboradores CD4 que a través de la secreción de citocinas favorecen la quimiotaxis y activación de eosinófilos y la síntesis de IgE, perpetuando la respuesta al alergeno. Incluso en sus estadios más iniciales, el asma se caracteriza por el infiltrado de la mucosa por eosinófilos activados ${ }^{7}$. Estas alteraciones también se han evidenciado, aunque en menor grado, en las muestras de biopsia bronquial ${ }^{8,9}$ y esputo inducido ${ }^{10-12}$ de sujetos con rinitis alérgica. Ocurre del mismo modo a la inversa, ya que en asmáticos sin síntomas nasales se aprecia infiltrado eosinofílico de la mucosa nasal ${ }^{13}$.

Probablemente como consecuencia de la cronificación de la inflamación en la pared bronquial, se producen una serie de cambios, irreversibles en su mayor parte, de la arquitectura de la vía aérea: descamación epitelial, fibrosis subepitelial, proliferación de fibroblastos y aumento de la masa de músculo liso. Este fenómeno, conocido como remodelado de la vía aérea, se aprecia incluso en los casos más leves de asma ${ }^{7}$, pero también en los sujetos con rinitis aislada, que presentan depósitos de colágeno bajo la membrana basal ${ }^{9,14}$. Una de las consecuencias del remodelado es la hiperreactividad bronquial $(\mathrm{HRB})^{7}$ o el aumento de la sensibilidad frente a estímulos broncoconstrictores, que además de ser una de las características centrales del asma, se observa en un considerable número de individuos con rinitis alérgica, en especial cuando ésta es perenne $\mathrm{e}^{15,16}$.

\section{Etiología}

Los alergenos y algunas sustancias ocupacionales son los únicos agentes que hasta el momento, han demostrado su capacidad para iniciar y mantener la reacción inmunoinflamatoria propia del asma $^{17}$. En asmáticos susceptibles, la inhalación del alergeno, ya sea de forma natural o provocada (provocación bronquial con alergeno) induce un descenso inmediato de la función pulmonar (respuesta inmediata), seguido al cabo de 6 a 8 horas de un incremento transitorio de la inflamación eosinofílica bronquial y de la HRB (respuesta tardía). En un número considerable de pacientes alérgicos con rinitis y sin evidencia clínica de asma, la administración del alergeno, ya sea mediante provocación bronquial ${ }^{18,19}$ o incluso a través de su instilación nasal ${ }^{20}$, induce una respuesta inflamatoria y funcional, similar aunque de menor intensidad a la observada en asmáticos.

Por último, la rinitis y el asma alérgicos comparten un fondo de atopia común que podría estar relacionado con genes situados en 13 cromosomas diferentes, aunque algunos alelos de los cromosomas 5 y 11 son los que muestran una mayor asociación con los valores de IgE, el grado de HRB y el fenotipo de asma y/o rinitis ${ }^{21}$.

\section{Terapéutica}

El control subóptimo de la rinitis, ya sea como consecuencia de la pérdida de las funciones protectoras y homeostáticas de las fosas nasales, del goteo postnasal de mediadores o de la puesta en funcionamiento de mecanismos neurogénicos, empeora el asma ${ }^{4}$. De acuerdo con ello, el tratamiento de la rinitis alérgica, en especial con corticoides tópicos nasales, reduce los síntomas bronquiales ${ }^{22}$, la $\mathrm{HRB}^{22}$ y las exacerbaciones de asma ${ }^{23}$. Resulta especialmente llamativo el caso del síndrome de Samter en el que la polipectomía mejora los síntomas bronquiales, contemplándose en ocasiones como tratamiento alternativo del asma ${ }^{24}$. A la inversa, el tratamiento con corticoides tópicos bronquia- 
les en sujetos con rinitis alérgica sin asma, reduce los síntomas nasales y la inflamación eosinofílica en la mucosa nasal ${ }^{25}$.

Por otra parte, los tratamientos que muestran mayor actividad sobre las dos enfermedades tienden a ser sistémicos, de modo que las medidas de control ambiental de la exposición alergénica ${ }^{26}$ y la vacunación con alergeno ${ }^{27}$ además de mejorar los síntomas de rinitis y asma, modifican la historia natural de la enfermedad previniendo su progresión hacia el desarrollo de asma y de nuevas sensibilizaciones ${ }^{28}$. El tratamiento con antileucotrieno $\mathrm{s}^{29} \mathrm{o}$ anticuerpos recombinantes humanizados anti$\operatorname{IgE}^{30}$ es efectivo también para ambas entidades, lo que sugiere de nuevo una interrelación entre rinitis y asma.

\section{CONSIDERACIONES FINALES}

A partir de estos datos, algunos autores consideran a la enfermedad alérgica respiratoria como un espectro continuo de manifestaciones clínicas, con pacientes con síntomas nasales exclusivamente en un extremo, y pacientes con asma y rinitis manifiestas en el otro, pasando por los individuos que únicamente presentan síntomas nasales y en los que se aprecia inflamación de vías aéreas inferiores e $\mathrm{HRB}^{31}$ Este concepto de "vía aérea única" no explica sin embargo, por qué el grupo intermedio de pacientes, con inflamación eosinofílica en vías aéreas inferiores e HRB no presenta síntomas de asma, lo que podría atribuirse a una pobre percepción de los síntomas de obstrucción al flujo aéreo $^{32}$. No obstante, otras entidades no alérgicas como la helmintiasis, la neumonía eosinófila o el síndrome hiper-IgE entre otras, cursan también con eosinofilia pulmonar y valores elevados de IgE sin que se aprecien datos clínicos ni funcionales sugestivos de asma. Todo ello sugiere que deben intervenir otros factores ya sean de tipo genético o propios de las diferencias intrínsecas en la arquitectura y dinámica bronquial, que determinen que un sujeto dado, en un contexto adecuado de sensibilización alérgica, desarrolle o no síntomas de asma.

Por otra parte, la respuesta inmunológica al alergeno se desarrolla en la vía aérea, pero también en otros órganos como la piel o la sangre periférica, lo que constituye el fundamento de las pruebas diagnósticas empleadas en alergología. Especialmente en los sujetos que desarrollan respuestas tardías, la inhalación del alergeno induce en la médula ósea incremento del número de células precursoras de eosinófilos y de los niveles de citocinas que promueven su proliferación y diferenciación (interleucina-5, eotaxina) ${ }^{33}$. Los sujetos con rinitis e HRB a metacolina (agonista colinérgico) muestran también hiperreactividad colinérgica en otros órganos como la piel o el intestino ${ }^{34}$. Por otra parte, algunos pacientes con rinitis y asma, alérgicos a ácaros, desarrollan episodios de anafilaxia secundarios a la ingesta de alimentos elaborados con harinas contaminadas por ácaros ${ }^{35}$. Todo ello sugiere que la rinitis y el asma son las manifestaciones respiratorias de una enfermedad sistémica caracterizada por la respuesta inmunológica anómala frente al alergeno. El hecho de que sean las líneas terapéuticas aplicadas de forma sistémica (evitación alergénica, vacunación con alergeno, anticuerpos anti-IgE o antileucotrienos) las más eficaces para el tratamiento concomitante de las dos enfermedades ${ }^{28.30}$ apoya esta hipótesis.

\section{IMPLICACIONES PRÁCTICAS}

Ya que la mayor intensidad de los síntomas de uno de los órganos puede determinar que el individuo subestime los del otro $^{36}$, resulta imprescindible descartar mediante anamnesis, exploración física y pruebas complementarias, la coexistencia de rinitis o asma en cualquier paciente que presente síntomas aislados de una de ellas. Ello es debido a que,

1- El diagnóstico y tratamiento precoz del asma de reciente inicio puede retrasar e incluso impedir el remodelado irreversible de la vía aérea y por tanto la cronificación del asma ${ }^{37}$

2- El diagnóstico y tratamiento de la posible patología nasal contribuirá a optimizar el control de la enfermedad asmática.

3- El tratamiento etiológico de los síntomas nasales (control ambiental y vacunación con alergeno), además de mejorar 
las dos enfermedades, contribuye a modificar la historia natural de la enfermedad alérgica, previniendo nuevas sensibilizaciones y el desarrollo de asma ${ }^{26,28}$.

En segundo lugar, parece importante que el objetivo del tratamiento sea, además de la mejoría sintomática del paciente, el control de la respuesta inflamatoria subyacente y de la respuesta inmunológica sistémica frente al alergeno. Los corticoides, a través de la inhibición de factores de transcripción, son fármacos de elevada potencia en el control de la respuesta inmunoalérgica tanto del asma como de la rinitis. La disponibilidad de corticoides por vía inhalada ha resuelto parcialmente sus efectos adversos sistémicos que sin embargo pueden reaparecer a dosis elevadas $^{38}$. Esto unido a que actúan de manera inespecífica sobre la respuesta inmunoalérgica, no son capaces de alterar el curso natural de la enfermedad que reaparece poco después de su suspensión y, no revierten el proceso de remodelado una vez establecido ${ }^{38}$, hace necesaria la investigación de nuevos fármacos que, con un perfil de seguridad superior, permitan de forma más selectiva controlar la enfermedad alérgica respiratoria. Sería además aconsejable disponer de un fármaco que permitiera tratar al mismo tiempo la rinitis y el asma, lo que aumentaría la adhesión del paciente al tratamiento. Por el momento, las medidas de control ambiental y la vacunación con alergeno, son las únicas aproximaciones terapéuticas que han demostrado cierta capacidad para controlar y modificar el curso de la enfermedad alérgica. En los últimos años se están desarrollando diversas líneas de investigación con el fin de conseguir el fármaco ideal para el tratamiento integral del paciente alérgico. Entre ellos destacan algunos ya comercializados (antileucotrienos, anticuerpos monoclonales anti-IgE, antihistamínicos de nueva generación) y otros en fase de estudio (inhibidores selectivos de la fosfodiesterasa o de las citocinas Th2 e inhibidores de moléculas de adhesión o de las quimiocinas $)^{38}$

\section{BIBLIOGRAFÍA}

1. Simons F. Ancestors of Allergy. New York: Global Medical Communications. 1994.
2. Guidelines for the diagnosis and management of asthma. National Heart, Lung, and Blood Institute. National Asthma Education Program. Expert Panel Report. J Allergy Clin Immunol 1991; 88: 425-534.

3. International Consensus Report on Diagnosis and Management of Rhinitis. Management working Group. Allergy 1994; 49: 1-34.

4. Barnes P, WoOlcock A. Difficult asthma. Eur Respir J 1998; 12:1209-1218.

5. Kapsali T, Horowitz E, Diemer F, Togias A Rhinitis is ubiquitous in allergic asthmatics. J Allergy Clin Immunol 1997; 99: 138.

6. Leynaert B, Bousouet J, Neukirch C, Liard R, NEUKIRCH F. Perennial rhinitis: An independent risk factor for asthma in nonatopic subjects: results from the European Community Respiratory Health Survey. J Allergy Clin Immunol 1999; 104: 301-304.

7. JeFFERY PK, WARDLAW AJ, NELSON FC, COLLINS JV, KAY AB. Bronchial biopsies in asthma. An ultrastructural, quantitative study and correlation with hyperreactivity. Am Rev Respir Dis 1989; 140: 1745-1753.

8. DJUKANOVIC R, LaI CK, WILSON JW, BRITTEN KM WILSON SJ, Roche WR et al. Bronchial mucosal manifestations of atopy: a comparison of markers of inflammation between atopic asthmatics, atopic nonasthmatics and healthy controls. Eur Respir J 1992; 5: 538544

9. Boulet LP, Laviolette M, Turcotte H, Cartier A, Dugas M, Malo JL et al. Bronchial subepithelial fibrosis correlates with airway responsiveness to methacholine. Chest 1997; 112: 45-52.

10. Foresi A, Leone C, Pelucchi A, Mastropasoua B, ChetTA A, D' IPPOLITo R. Eosinophils, mast cells, and basophils in induced sputum from patients with seasonal allergic rhinitis and perennial asthma: relationship to methacholine responsiveness. J Allergy Clin Immunol 1997; 100: 58-64.

11. Polosa R, Ciamarra I, Mangano G, Prosperini G Pistorio MP, VANCHERI C et al. Bronchial hyperresponsiveness and airway inflammation markers in nonasthmatics with allergic rhinitis. Eur Respir J 2000; 15: 30-35.

12. Alvarez MJ, Olaguibel JM, Garcia BE, RoDRIOUEZ A, TABAR AI, URBIOLA E. Airway inflammation in asthma and perennial allergic rhinitis. Relationship with nonspecific bronchial responsiveness and maximal airway narrowing [In Process Citation]. Allergy 2000; 55: 355-362. 
13. GAGA M, LAMBROU P, PAPAGEORGIOU N Eosinophils are a feature of upper and lower airway pathology in non-atopic asthma irrespective of the presence of rhinitis. Clin Exp Allergy 2000; 30: 663-669.

14. BOUSQuet J, JEFFERY P, BusSE W, JOHNSON M Vignola A. Asthma: from bronchoespasm to airway remodelling. Am J Respir Crit Care Med 2000; 161: 1720-1745.

15. Sterk PJ, Fabbri LM, Quanjer PH, Cockcroft DW, O'Byrne PM, ANDERSON SD et al. Airway responsiveness. Standardized challenge testing with pharmacological, physical and sensitizing stimuli in adults. Report Working Party Standardization of Lung Function Tests, European Community for Steel and Coal. Official Statement of the European Respiratory Society. Eur Respir J Suppl 1993, 16: 53-83.

16. PRIETo L, Marin J. Measurement of response to methacholine inhalation challenge in asthma and allergic rhinitis. PC20 or PC35FEV1? Allergol Immunopathol 1990; 18 285-289.

17. PRIETO L. Alergenos y asma: algunos conceptos han cambiado. Med Clin (Barc) 1994; 102: 376-379.

18. Bonavia M, Crimi E, Quaglia A, Brusasco V. Comparison of early and late asthmatic responses between patients with allergic rhinitis and mild asthma. Eur Respir J 1996 9: 905-909.

19. Alvarez MJ, Olaguibel JM, Garcia Be, Tabar AI URBIOLA E. Comparison of allergen-induced changes in bronchial hyperresponsiveness and airway inflammation between mildly allergic asthma patients and allergic rhinitis patients. Allergy 2000; 55: 531-539.

20. Beeh KM, Beier J, Kornmann O, Meier C, TAEumer T, BuHL R. A single nasal allergen challenge increases induced sputum inflammatory markers in non-asthmatic subjects with seasonal allergic rhinitis: correlation with plasma interleukin-5. Clin Exp Allergy 2003; 33: 475-482.

21. HALL I. Genetic factors in asthma severity. Clin Exp Allergy 1998; 28: 16-20.

22. WATSON WT, BECKER AB, SimONS FE. Treatment of allergic rhinitis with intranasal corticosteroids in patients with mild asthma: effect on lower airway responsiveness. J Allergy Clin Immunol 1993, 91: 97-101.

23. Adams R, Fuhlbrigge A, Finkelstein J, Weiss S. Intranasal steroids and the risk of emergency department visits for asthma. J Allergy Clin Immunol 2002; 109: 636-642.
24. ShUAIB NASSER S, LEE T. Inflammatory mechanisms of aspirin-sensitive asthma. In: Holgate, ST, Busse WW. En: Inflammatory mechanisms in asthma. Marcel Dekker Inc 1998: 823-844.

25. GReifF L, ANDERSSON M, SvensSon C. Effects of oral inhaled budesonide in seasonal allergic rhinitis. Eur Resp J 1998; 11:1268-1273.

26. Platts-Mills T, Vaughan J, Carter $\mathrm{M}$, WOODFOLK J. The reole of intervention in established allergy: evoidance of indoor allergens in the treatment of chronic allergic disease. J Allergy Clin Immunol 2000; 106:787-804.

27. Bousouet J, Lockey R, Malding H. Who position paper. Allergen immunotherapy: therapeutic vacunes for allergic diseases Eur J Respir 1998; 53: 44.

28. Des Roches A, Paradis L, Menardo J, Bouges S DAURES J, BousQueT J. Immunotherapy with a standardized Dermatophagoides pteronyssinus extract. VI. Specific immunotherapy prevents the onset of new sensitizations in children. J Allergy Clin Immunol 1997; 99: 450-453.

29. Wood R, Phipatanakul W, Conover-Walker M Kesvanathan J, Sweitzer D, Eggleston P. A randomized double-blinded placebo controlled trial of zafirlukast on upper and lower respiratory responses to cat challenge. J Allergy Clin Immunol 2000; 105: 367.

30. Casale T, Condemi J, Miller S, Fick R, McAlary M, FOWLER TAYLOR A. RhuMab-E25 in the treatment of seasonal allergic rhinitis (SAR) Ann Allergy Asthma Immunol 1999; 82: 75.

31. TogíAS A. Mechanisms of nose-lung interaction. Allergy 1999; 54: 94-105.

32. Salome CM, Xuan W, Gray EJ, Belooussova E, PEAT JK. Perception of airway narrowing in a general population sample. Eur Respir J 1997; 10: 1052-1058

33. Wood LJ, Sehmi R, Dorman S, Hamid Q, Tulic MK, WATSON RM, et al. Allergen-induced increases in bone marrow $\mathrm{T}$ lymphocytes and interleukin-5 expression in subjects with asthma. Am J Respir Crit Care Med 2002; 166 883-889.

34. CASAlE T, BaraniuK J. Neurogenic control of inflammation and airway function. En: Middleton: Principles and Practice, 5th ed Middleton E, Reed CE, Ellis EF, Adkinson NF Yunginger, JW and Busse WW, eds. MosbyYear book, St Louis, MO 1998: 183-203.

35. Blanco C, Castillo R, Ortega N, Alvarez M ARTEAGA C, BARBER D et al. Asthma due to the 
ingestion of contaminated flour. J Investig Allergol Clin Immunol 1997; 7: 323-4.

36. LeynaERT B, NEukiRCH C, Liard R, Bousouet J, NEURKIRCH F. Quality of life in rhinitis and asthma: a population based study of young adults. Am J Respir Crit Care Med 2000; 162 . 1391-1396.

37. LAITINEN LA, LAitinEN A, HAAHTEla T. A comparative study of the effects of an inhaled corticosteroid, budesonide, and a beta 2-agonist, terbutaline, on airway inflammation in newly diagnosed asthma: a randomized, double-blind, parallel-group controlled trial. J Allergy Clin Immunol 1992; 90: 32-42.

38. BARNES P. New directions in allergic diseases: mechanism-based antiinflamatory therapies. J Allergy Clin Immunol 2000; 106: 5-16. 